\title{
FOURIER ANALYSIS OF THE INTRA-AORTIC BALLOON PUMP
}

Michael Poullis, Bsc(Hons), MBBS, FRCS(Eng), London, United Kingdom

In patients with an intra-aortic balloon pump (IABP), maximum blood pressure augmentation occurs with the balloon set to $1: 1$ mode. However, there is a subset of patients in whom better blood pressure augmentation is obtained when the balloon is set to $1: 2$ or even 1:3.

Systemic vascular resistance is a commonly used term, but it is misleading because it implies that it is a constant, regardless of heart rate. In practice this is not true, hence the adoption of the term impedance. It has been shown that, when the

From the Department of Cardiothoracic Surgery, National Heart and Lung Institute, Imperial College of Science, Technology and Medicine, Hammersmith Hospital, London, United Kingdom.

Received for publication Sept 15, 1998; accepted for publication Nov 5, 1998.

J Thorac Cardiovasc Surg 1999;117:827-8

Copyright $\odot 1999$ by Mosby, Inc.

$0022-5223 / 99 \$ 8.00+0 \quad \mathbf{1 2 / 5 4 / 9 5 7 5 7}$ heart and vasculature are matched for impedance, maximum systolic blood pressure results. ${ }^{1,2}$ The impedance of the vasculature is dependent not just on the heart rate but also on the vascular compliance and the viscosity of the blood.

The arterial waveforms of patients are conventionally viewed with time on the horizontal axis, the time domain. If the arterial waveforms are viewed with the frequency components that make up the arterial waveforms on the horizontal axis, then this is called the frequency domain.

Frequency domain analysis takes into account systemic vascular impedance, blood viscosity, vascular compliance, blood volume, and heart rate because these determine the shape of the arterial pressure waveform. The technique of converting an arterial waveform in the time domain to 1 in the frequency domain is called Fourier analysis, the mathematics of which is beyond the scope of this article.

Matching the heart rate to the fundamental frequencies obtained from frequency domain analysis of the arterial 

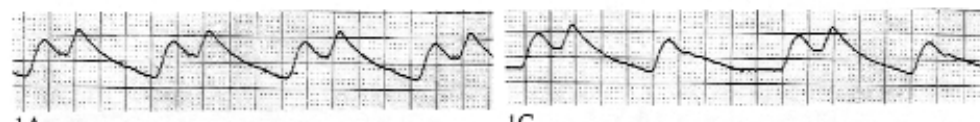

$1 \mathrm{~A}$

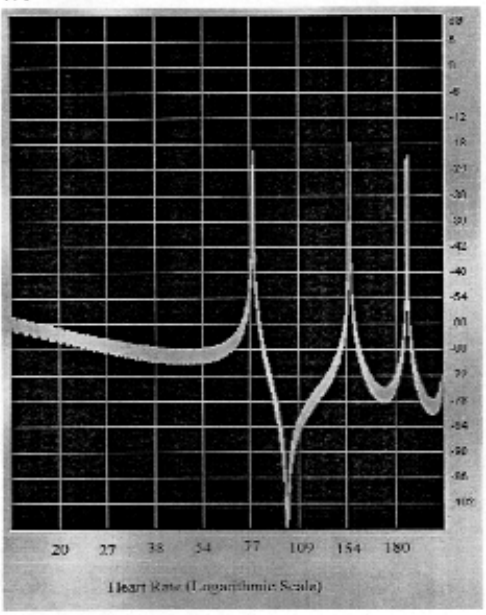

IB
IC

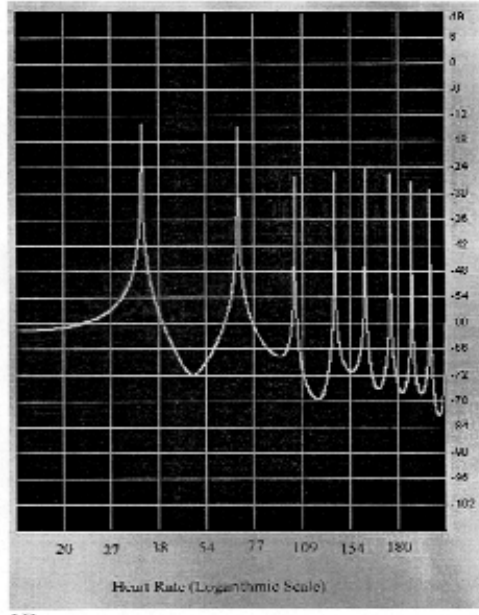

11)
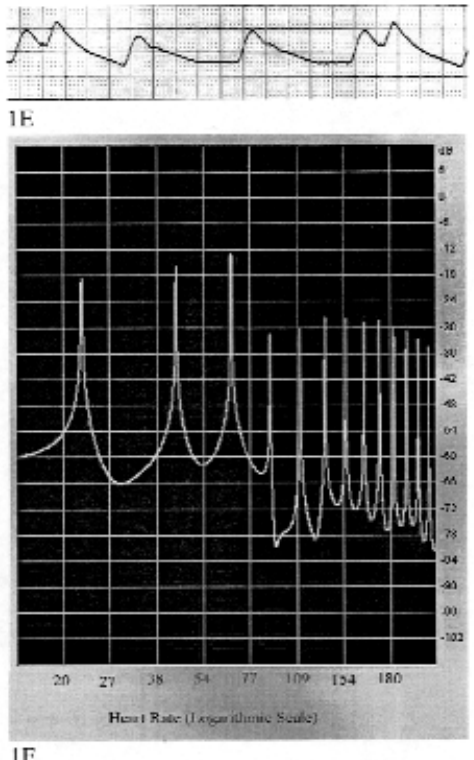

TIME

DOMAIN ANAI.YSIS FRHQUFNCY DOMAIN ANALYSIS

Fig 1. The intra-atrial tracings from a patient with a balloon pump inserted that is operating in 1:1 mode (A), 1:2 mode $(\mathbf{C})$, and 1:3 mode $(\mathbf{E})$ are shown (time domain analysis). Fast Fourier transforms of IABP intra-arterial trace for 1:1 mode $(\mathbf{B})$, 1:2 mode $(\mathbf{D})$, and 1:3 mode $(\mathbf{F})$ are shown (frequency domain analysis). Vertical scale is in decibels. The sharp upward spikes represent the component frequencies of the arterial waveform, and it is these frequencies for which the impedance should be matched.

waveforms would have major benefits for patients because of the impedance matching that would result. ${ }^{3}$

The typical intra-arterial tracing from a patient with a balloon pump inserted that is operating in $1: 1,1: 2$, and $1: 3$ are shown in Fig 1, $A, C$, and $E$, respectively (time domain). Fast Fourier analysis (Syntrillium Software Corporation, Phoenix, Ariz) was used to convert the time domain data into the frequency domain.

Fig $1, B, D$, and $F$, shows the frequency spectra obtained from analyzing the intra-arterial pressure traces with a balloon pump inserted that is operating in $1: 1,1: 2$, and $1: 3$ mode, respectively (frequency domain). The frequency has been expressed as heart rate normalized to a rate of 60 beats/min to aid interpretation. The term decibel $(\mathrm{dB})$ is the logarithm to the base 10 of a ratio; thus the vertical scales of the graphs are basically logarithmic scales. From Fig 1, $B, D$, and $F$, it can be seen that there is at least a $30 \mathrm{~dB}$ fall off (which equates to a factor of about 1000) on either side of the frequency peaks, which would relate to a highly significant mismatch $(P<.001)$. Optimal heart rate with the IABP in $1: 1$ mode was 77 beats/min, in 1:2 mode was 69, 101, and 135 beats/min, and in 1:3 mode $66,89,111$, and 134 beats/min.

Intraoperative pacing wire placement is routine, especially in high-risk patients. This makes manipulation of the heart rate very easy. Patients have been paced often at a rate that has been found to be their optimal rate, that is, matched impedance, before a decision has been made to insert the IABP.

The data provided proves that there are certain specific optimal rates at which the heart will provide a maximal sys- tolic blood pressure, because of impedance matching. This rate will vary depending on whether the IABP is operating in $1: 1,1: 2$, or $1: 3$ mode, because of the different associated harmonic spectra. Multiple potential optimal heart rates occur in 1:2 mode and in 1:3 mode because of harmonic overtones, which are created by the IABP.

Only the case of a patient in sinus rhythm has been considered, but the technique remains valid if the rhythm is regular (this includes atrial flutter and ventricular tachycardia). This means that patients with atrial fibrillation should be ventricular overdrive paced to artificially create a regular rhythm. Irregular waveforms and waveforms with ectopics are unsuitable for this method of analysis.

Thus instead of just adjusting the inflation and deflation times and leaving the heart rate alone, as long as it falls within the physiologic range, we have shown that the heart rate can have a major impact on the functioning, through systolic blood pressure augmentation, of an IABP because of the heart rate dependence of impedance matching.

\section{REFERENCES}

1. Little WC, Cheng CP. Left ventricular-arterial coupling in conscious dogs. Am J Physiol 1991;261(1 Pt 2):H70-6.

2. Burkhoff D, Sagawa K. Ventricular efficiency predicted by an analytical model. Am J Physiol 1986;250(6 Pt 2):R1021-7.

3. Ross J Jr. Afterload mismatch and preload reserve: a conceptual framework for the analysis of ventricular function. Circ Res 1974;35;117-26. 